Pflegeberufsgesetz

\title{
Institut empfiehlt umfassende Evaluation
}

— Das Deutsche Institut für angewandte Pflegeforschung (dip) sieht im Referentenentwurf des Pflegeberufsgesetzes historische Chancen. Direktor Prof. Frank Weidner: „Mit der Generalisierung sind nun auch der gesetzliche Einstieg in die grundständige Akademisierung und erstmals vorbehaltene
Aufgaben für die Pflege vorgesehen." Zugleich plädiert er für eine gründliche Evaluation. Die Zusammenführung der bislang nebeneinander bestehenden drei Pflegeausbildungen (Altenpflege, Kinderkrankenpflege und Krankenpflege) zu einem neuen Beruf und die Einführung grundstän-

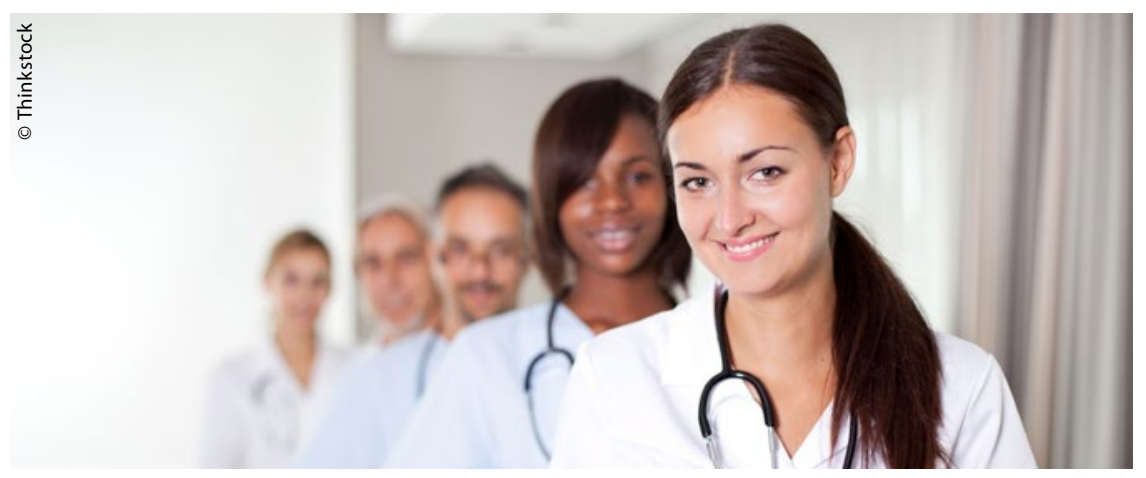

\section{Errichtungsausschuss ernannt}

\section{Pflegekammer Schleswig-Holstein}

— Die Pflegekammer in Schleswig-Holstein nimmt Form an. Sozialministerin Kristin Alheit hat die 13 Mitglieder des Errichtungsausschusses der Pflegeberufekammer ernannt. Es ist nun Aufgabe der ehrenamtlichen Mitglieder des Errichtungsausschusses die erste Kammerwahl zu organisieren und alle Mitglieder des Pflegeberufes zu

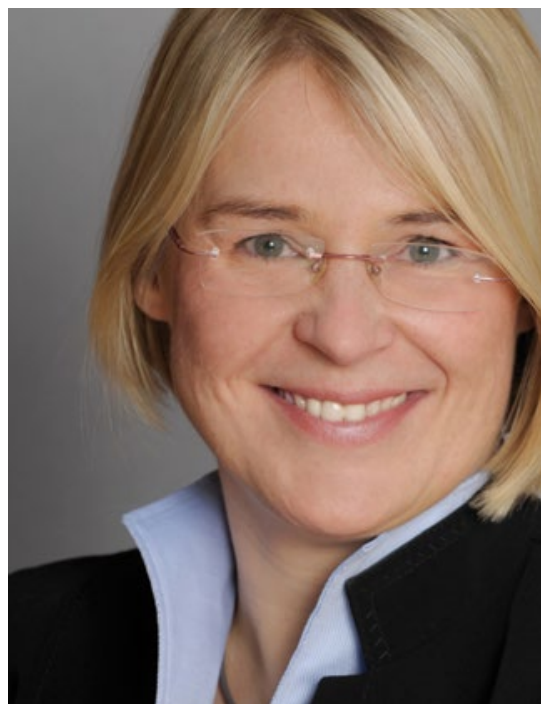

registrieren. „Die Pflegeberufekammer ist eine starke Basis für eine gleichwertige Anerkennung der Pflegeberufe im Gesundheitswesen. Dies ist auch ein wichtiger Beitrag zur Stärkung der Pflege insgesamt. Mit dem heutigen Tag kommen wir der Errichtung in Schleswig-Holstein einen wichtigen Schritt näher", erklärt die Sozialministerin. Das entsprechende Gesetz zur „Errichtung einer Kammer für die Heilberufe in der Pflege" wurde am 15. Juli 2015 vom Landtag Schleswig-Holstein verabschiedet. Schleswig-Holstein gehört damit neben Rheinland-Pfalz zu den ersten Bundesländern, das eine Pflegeberufekammer errichtet. Auch in Mecklenburg-Vorpommern fordern die beruflich Pflegenden eine Pflegekammer. Ulla Rose, Geschäftsführerin DBfK Nordost, appelliert an die Regierung, dem Land Rheinland-Pfalz nachzueifern: „Nach dem dortigen Spitzenwert von 75\% Zustimmung wurde umgehend begonnen, den Willen der Berufsgruppe umzusetzen. Das fordern wir auch von der Koalition in Mecklenburg-Vorpommern." diger Pflegestudiengänge folgen in der Konsequenz dem aktuellen Forschungsstand, stellt Weidner fest. Zugleich warnt er davor, diese Reform jetzt durch Partikularinteressen zu zerreden. Weidner weist darauf hin, dass „eine Reform, die wirklich etwas bewegen will, neben den Chancen immer auch offene Fragen mit sich bringt, sonst wär sie ja keine Reform". So gäbe es noch Fragen, die sich auf die zukünftige Entwicklung der Zahlen an Auszubildenden und Studierenden, auf die Umsetzung der praktischen Ausbildung oder auf den Übergang der Absolventen in das Beschäftigungssystem beziehen. Daher wird vom dip begrüßt, dass der Referentenentwurf bereits Schritte zur Überprüfung vorsieht. Weidner empfiehlt, noch offene Fragen, die aus dem Bauch heraus nicht zu beantworten seien, in eine umfassende, mindestens fünfährige Begleitforschung und Evaluation aufzunehmen.

www.dip.de

\section{Maßnahmenkatalog \\ Demenzfreundliches Saarland}

— Sozialministerin Monika Bachmann hat gemeinsam mit dem iso-Institut, der Landesfachstelle Demenz und weiteren regionalen Akteuren den ersten saarländischen Demenzplan der Öffentlichkeit vorgestellt. 29 konkrete Maßnahmen wurden in vier verschiedenen Handlungsfeldern vereinbart.

Der Plan soll dazu beitragen, die Betroffenen so lange wie möglich am normalen Leben teilhaben zu lassen und die pflegenden Angehörigen zu entlasten. Außerdem gehe es darum, die Öffentlichkeit zu sensibilisieren und neue Fortbildungs- und Informationsangebote für verschiedene Zielgruppen zu entwickeln. Besondere Bedeutung komme der weiteren Vernetzung der vorhandenen Versorgung im ambulanten, teilstationären und stationären Bereich zu. Die Maßnahmen gliedern sich in die vier Felder. Mit einem landesweiten Demenzplan nimmt das Saarland neben Bayern und Schleswig-Holstein bundesweit eine Vorreiterrolle ein.

www.saarland.de/publikationen-soziales.htm 